\title{
THE ELASTIC FIELDS OF A LINE FORCE OR DISLOCATION IN AN ANISOTROPIC WEDGE
}

\author{
KUANG-CHONG WU \\ Institute of Applied Mechanics, National Taiwan University, Taipei, Taiwan
}

(Received 11 September 1996; in revised form 16 July 1997)

\begin{abstract}
An elasticity analysis for a line force or dislocation in an infinite anisotropic wedge is presented. A solution in integral form is derived by the Mellin integral transform method. It is shown that the solution integral can be expanded into eigenfunction series by residue theorem. The asymptotic fields as the radial distance approaches zero or infinity are discussed. (C) 1998 Elsevier Science Ltd. All rights reserved.
\end{abstract}

\section{INTRODUCTION}

One of the basic solutions in elasticity is the fields produced by a line force or a line dislocation. The solutions due to these line singularities are important in constructing integral equations for boundary value problems. For example, the conventional boundary integral equations are based on the line force solution (Rizzo, 1967). Some new boundary integral equations are established using both line force and dislocation solutions (Wu et $a l ., 1992)$. The dislocation solutio is also widely used to set up integral equations for solving crack problems.

The solution for a line dislocation in an infinite anisotropic medium has been obtained by Eshelby et al. (1953), Stroh (1958), Willis (1970), Malen and Lothe (1970), and Malen (1971). Malen's (1971) treatment also includes a line force. The solution for an anisotropic half-space subject to a line force and/or a line dislocation has been considered by Head (1953, 1965), Chou (1966), Tucker (1969), Willis (1970), Barnett and Lothe (1975). The problem where the force is at the tip of an anisotropic wedge was studied by Barnett and Lothe (1975). Similar plane strain solutions for anisotropic wedges subject to boundary traction was provided by Bogy (1972) with focus on stress singularities at apex. An anisotropic wedge under a concentrated couple at the tip was investigated by Ting (1988) and Hwu and Ting (1990). The near-tip fields of a wedge with a line force or dislocation were derived by Wu and Chang (1993).

In this paper a line force or a line dislocation in an anisotropic wedge is considered. Stroh's formalism for anisotropic elasticity and Mellin integral transform are employed to derive the full solution in a compact and explicit form. The solution is expressed either in terms of an integral or eigenfunction series by evaluating the residues of the integral.

The plan of the paper is as follows. In Section 2 the basic equations are given. The solution in integral form is derived in Section 3. The eigenfunction series solution is derived in Section 4. Asymptotic fields are discussed in Section 5. Finally some remarks are made.

\section{BASIC EQUATIONS}

In the following discussion vectors or matrices are denoted by bold-faced letters. A subscript comma denotes partial differentiation and repeated Latin indices imply summation from 1-3 unless noted otherwise. Summation over Greek indices is indicated explicitly.

For a homogeneous anisotropic body, the generalized Hookes law and the equations of equilibrium are given as 


$$
\begin{gathered}
\text { K.-C. Wu } \\
\sigma_{i j}=C_{i j k l} u_{k, l} \\
\sigma_{i j, j}=0
\end{gathered}
$$

where $u_{k}$ and $\sigma_{i j}$ is the displacement and stress component, respectively, $C_{i j k l}$ is the component of the elasticity tensor. If the displacement $\mathbf{u}$ depends only on $x_{1}$ and $x_{2}$, so does the corresponding stress tensor $\sigma$. In view of eqn (2), a stress function $\phi$ can be introduced such that

$$
\sigma_{i 1}=-\phi_{i, 2}, \quad \sigma_{i 2}=\phi_{i, 1}, \quad i=1,2,3
$$

Substitution of eqn (3) into eqn (1) yields

$$
\begin{aligned}
\mathbf{Q} \mathbf{u}_{, 1}+\mathbf{R} \mathbf{u}_{, 2} & =-\phi_{, 2} \\
\mathbf{R}^{T} \mathbf{u}_{, 1}+\mathbf{T} \mathbf{u}_{, 2} & =\phi_{, 1}
\end{aligned}
$$

where the components of the three by three matrices $\mathbf{Q}, \mathbf{R}$, and $\mathbf{T}$ are defined as

$$
\begin{aligned}
Q_{i k} & =C_{i 1 k 1} \\
R_{i k} & =C_{i 1 k 2} \\
T_{i k} & =C_{i 2 k 2}
\end{aligned}
$$

Equation (4) can be rewritten as (Chadwick and Smith, 1977)

$$
\mathbf{w}_{, 2}=\mathbf{N} \mathbf{w}_{, 1}
$$

where the six-dimensional vector $\mathbf{w}$ and six by six matrix $\mathbf{N}$ stand for

$$
\begin{gathered}
\mathbf{w}=\left[\begin{array}{l}
\mathbf{u} \\
\phi
\end{array}\right] \\
\mathbf{N}=\left[\begin{array}{ll}
\mathbf{N}_{1} & \mathbf{N}_{2} \\
\mathbf{N}_{3} & \mathbf{N}_{1}^{T}
\end{array}\right]
\end{gathered}
$$

and the three by three matrices $\mathbf{N}_{1}, \mathbf{N}_{2}$ and $\mathbf{N}_{3}$ are given by

$$
\begin{gathered}
\mathbf{N}_{1}=-\mathbf{T}^{-1} \mathbf{R}^{T} \\
\mathbf{N}_{2}=\mathbf{T}^{-1}=\mathbf{N}_{2}^{T} \\
\mathbf{N}_{3}=\mathbf{R T}^{-1} \mathbf{R}^{T}-\mathbf{Q}=\mathbf{N}_{3}^{T}
\end{gathered}
$$

In terms of polar coordinates $r$ and $\theta$ eqn (5) can be expressed as (Ting, 1996)

$$
\mathbf{w}_{, \theta}=r \mathbf{N}(\theta) \mathbf{w}_{, r}
$$

where

$$
\begin{aligned}
& \mathbf{N}(\theta)=\left[\begin{array}{ll}
\mathbf{N}_{1}(\theta) & \mathbf{N}_{2}(\theta) \\
\mathbf{N}_{3}(\theta) & \mathbf{N}_{1}(\theta)^{T}
\end{array}\right] \\
& \mathbf{N}_{1}(\theta)=-\mathbf{T}^{-1}(\theta) \mathbf{R}^{T}(\theta)
\end{aligned}
$$




$$
\begin{gathered}
\mathbf{N}_{2}(\theta)=\mathbf{T}^{-1}(\theta) \\
\mathbf{N}_{3}(\theta)=\mathbf{R}(\theta) \mathbf{T}^{-1}(\theta) \mathbf{R}^{T}(\theta)-\mathbf{Q}(\theta)
\end{gathered}
$$

and

$$
\begin{gathered}
Q_{i k}(\theta)=c_{i j k s} n_{j}(\theta) n_{s}(\theta) \\
R_{i k}(\theta)=c_{i j k s} n_{j}(\theta) m_{s}(\theta) \\
T_{i k}(\theta)=c_{i j k s} m_{j}(\theta) m_{s}(\theta) \\
\mathbf{n}^{T}(\theta)=[\cos \theta, \sin \theta, 0] \\
\mathbf{m}^{T}(\theta)=[-\sin \theta, \cos \theta, 0]
\end{gathered}
$$

Applying the Mellin transform to eqn (11) with respect to $r$, we get

$$
\tilde{\mathbf{w}}_{, \theta}=-\lambda \mathbf{N}(\theta) \tilde{\mathbf{w}}
$$

where

$$
\tilde{\mathbf{w}}(\theta)=\int_{0}^{\infty} \mathbf{w}(\theta, r) r^{\lambda-1} \mathrm{~d} r
$$

is the Mellin transform of $\mathbf{w}$.

Consider the following eigenvalue problem (Stroh, 1958):

$$
\mathbf{N} \xi=p \xi
$$

where $p$ is the eigenvalue and $\boldsymbol{\xi}$ is the right eigenvector of $\mathbf{N}$. Equation (23) provides six roots, $p_{\kappa}, \kappa=1, \ldots, 6$. Since $p_{\kappa}$ are complex if the strain energy is positive definite (Eshelby et al., 1953), we can let

$$
\begin{array}{ll}
p_{\kappa+3}=\bar{p}_{\kappa}, & \mathfrak{\Im}\left[p_{\kappa}\right]>0 \\
\xi_{\kappa+3}=\bar{\xi}_{\kappa}, & \kappa=1,2,3
\end{array}
$$

where $\mathfrak{I}$ denotes imaginary part and overbar stands for complex conjugate. Let $\boldsymbol{\eta}$ be the left eigenvector of $\mathbf{N}$ determined by

$$
\mathbf{N}^{T} \boldsymbol{\eta}=p \boldsymbol{\eta}
$$

Since

$$
\mathbf{N}^{T}=\mathbf{J N J}
$$

where the six by six matrix $\mathbf{J}$ is defined as

$$
\mathbf{J}=\left[\begin{array}{cc}
\mathbf{0} & \mathbf{I}_{3} \\
\mathbf{I}_{3} & \mathbf{0}
\end{array}\right]
$$

$\mathbf{I}_{3}$ being the three by three identity matrix. Comparing eqn (24) with eqn (23), we can set 


$$
\boldsymbol{\eta}=\mathbf{J} \boldsymbol{\xi}
$$

As the left eigenvector $\boldsymbol{\eta}$ and the right eigenvector $\boldsymbol{\xi}$ associated with different eigenvalue of $p$ are orthogonal to each other, we can normalize the eigenvectors such that

$$
\boldsymbol{\eta}_{\kappa}^{T} \xi_{\gamma}=\delta_{\kappa \gamma}
$$

where $\delta_{\kappa \gamma}$ is Kronecker's delta. The six by six identity matrix $\mathbf{I}_{6}$ can be expressed as the following spectral representation (Hoffman and Kunze, 1961) :

$$
\mathbf{I}_{6}=\sum_{\kappa=1}^{6} \boldsymbol{\xi}_{\kappa} \boldsymbol{\eta}_{\kappa}^{T}
$$

and the matrix $\mathbf{N}(\theta)$ in eqn (11) as (Ting, 1996)

$$
\mathbf{N}(\theta)=\sum_{\kappa=1}^{6} \frac{\zeta_{\kappa}(\theta)^{\prime}}{\zeta_{\kappa}(\theta)} \boldsymbol{\xi}_{\kappa} \boldsymbol{\eta}_{\kappa}^{T}
$$

where $\zeta_{\kappa}(\theta)=\cos \theta+p_{\kappa} \sin \theta$ and $\zeta_{\kappa}(\theta)^{\prime}$ is the derivative of $\zeta_{\kappa}(\theta)$ with respect to $\theta$. Equation (28) indicates that $\xi_{\kappa}$ and $\eta_{\kappa}$ are right and left eigenvectors, respectively, and $\zeta_{\kappa}(\theta)^{\prime} / \zeta_{\kappa}(\theta)$ is the corresponding eigenvalue of $\mathbf{N}(\theta)$. With eqn (28) into eqn (21), the general solution of $\tilde{\mathbf{w}}$ can be expressed as

$$
\tilde{\mathbf{w}}(\theta)=\mathbf{G}\left(\theta, \theta_{0} ; \lambda\right) \tilde{\mathbf{w}}\left(\theta_{0}\right)
$$

where the six by six matrix $\mathbf{G}$ is given by

$$
\mathbf{G}\left(\theta, \theta_{0} ; \lambda\right)=\sum_{\kappa=1}^{6}\left(\frac{\zeta_{\kappa}(\theta)}{\zeta_{\kappa}\left(\theta_{0}\right)}\right)^{-\lambda} \boldsymbol{\xi}_{\kappa} \boldsymbol{\eta}_{\kappa}^{T}
$$

and $\theta_{0}$ is an arbitrary angle.

The matrix $\mathbf{G}$ has the following properties :

$$
\begin{gathered}
\mathbf{G}\left(\theta_{0}, \theta_{0} ; \lambda\right)=\mathbf{G}\left(\theta, \theta_{0} ; 0\right)=\mathbf{I}_{6} \\
\mathbf{G}\left(\theta, \theta_{0} ; \lambda\right)=\mathbf{G}\left(\theta, \theta_{1} ; \lambda\right) \mathbf{G}\left(\theta_{1}, \theta_{0} ; \lambda\right) \\
\mathbf{G}\left(\theta, \theta_{0} ;-\lambda\right) \mathbf{G}\left(\theta, \theta_{0} ; \lambda\right)=\mathbf{I}_{6}
\end{gathered}
$$

If we let

$$
\boldsymbol{\xi}_{\kappa}=\left[\begin{array}{c}
\mathbf{A}_{\kappa} \\
\mathbf{B}_{\kappa}
\end{array}\right], \quad \boldsymbol{\eta}_{\kappa}=\mathbf{J} \boldsymbol{\xi}_{\kappa}=\left[\begin{array}{c}
\mathbf{B}_{\kappa} \\
\mathbf{A}_{\kappa}
\end{array}\right]
$$

where $\mathbf{A}_{\kappa}$ and $\mathbf{B}_{\kappa}$ are three-dimensional vectors, the matrix $\mathbf{G}$ can be partitioned into four three by three submatrices as follows,

$$
\mathbf{G}=\left[\begin{array}{cc}
\mathbf{G}_{1} & \mathbf{G}_{2} \\
\mathbf{G} & \mathbf{G}_{1}^{T}
\end{array}\right]
$$


where

$$
\begin{aligned}
& \mathbf{G}_{1}\left(\theta, \theta_{0} ; \lambda\right)=\sum_{\kappa=1}^{6}\left(\frac{\zeta_{\kappa}(\theta)}{\zeta_{\kappa}\left(\theta_{0}\right)}\right)^{-\lambda} \mathbf{A}_{\kappa} \mathbf{B}_{\kappa}^{T} \\
& \mathbf{G}_{2}\left(\theta, \theta_{0} ; \lambda\right)=\sum_{\kappa=1}^{6}\left(\frac{\zeta_{\kappa}(\theta)}{\zeta_{\kappa}\left(\theta_{0}\right)}\right)^{-\lambda} \mathbf{A}_{\kappa} \mathbf{A}_{\kappa}^{T} \\
& \mathbf{G}_{3}\left(\theta, \theta_{0} ; \lambda\right)=\sum_{\kappa=1}^{6}\left(\frac{\zeta_{\kappa}(\theta)}{\zeta_{\kappa}\left(\theta_{0}\right)}\right)^{-\lambda} \mathbf{B}_{\kappa} \mathbf{B}_{\kappa}^{T}
\end{aligned}
$$

Some identities which will be useful later are listed here (Hwu and Ting, 1990):

$$
\sum_{\kappa=1}^{6}\left(\frac{\zeta_{\kappa}(\theta)}{\zeta_{\kappa}\left(\theta_{0}\right)}\right)^{m} \log \left(\frac{\zeta_{\kappa}(\theta)}{\zeta_{\kappa}\left(\theta_{0}\right)}\right)^{n} \boldsymbol{\xi}_{\kappa} \boldsymbol{\eta}_{\kappa}^{T}=\mathbf{N}^{m}\left(\theta, \theta_{0}\right)\left(\pi \tilde{\mathbf{N}}\left(\theta, \theta_{0}\right)\right)^{n}
$$

where

$$
\begin{gathered}
\mathbf{N}\left(\theta, \theta_{0}\right)=\cos \left(\theta-\theta_{0}\right) \mathbf{I}_{6}+\sin \left(\theta-\theta_{0}\right) \mathbf{N}\left(\theta_{0}\right) \\
\tilde{\mathbf{N}}\left(\theta, \theta_{0}\right)=\frac{1}{\pi} \int_{\theta_{0}}^{\theta} \mathbf{N}(\omega) \mathrm{d} \omega
\end{gathered}
$$

In eqn (39) $m$ and $n$ are arbitrary integers. The matrix $\mathbf{N}\left(\theta, \theta_{0}\right)$ has the following properties :

$$
\mathbf{N}_{1}\left(\theta, \theta_{0}\right) \mathbf{m}\left(\theta_{0}\right)=\mathbf{m}(\theta), \quad \mathbf{N}_{3}\left(\theta, \theta_{0}\right) \mathbf{m}\left(\theta_{0}\right)=\mathbf{0}
$$

where $\mathbf{m}(\theta)$ is given by eqn (20).

\section{INTEGRAL SOLUTION}

Consider the wedge shown in Fig. 1. The $x_{1}$ axis is taken as the bisector of the wedge. The wedge is free of tractions at $\theta= \pm \alpha$. A line force $\mathbf{f}$ and a dislocation with Burgers vector $\mathbf{b}$ are located at $r=\rho$ and $\theta=\psi$. The boundary conditions at $\theta= \pm \alpha$ and the jump conditions at $\theta=\psi$ are described, respectively, by

$$
\begin{gathered}
\frac{\partial \phi}{\partial r}(r, \pm \alpha)=\mathbf{0} \\
\mathbf{w}\left(r, \psi^{+}\right)-\mathbf{w}\left(r, \psi^{-}\right)=-H(r-\rho) \mathbf{g}
\end{gathered}
$$

where $H$ is the Heaviside step function and $\mathbf{g}^{T}=\left[\mathbf{b}^{T}, \mathbf{f}^{T}\right]$. Applying the Mellin transform to eqns (43) and (44), we have

$$
\begin{aligned}
& (\lambda-1) \tilde{\phi}( \pm \alpha, \lambda-1)=\mathbf{0} \\
& \tilde{\mathbf{w}}\left(\psi^{+}\right)-\tilde{\mathbf{w}}\left(\psi^{-}\right)=\frac{\rho^{\lambda}}{\lambda} \mathbf{g}
\end{aligned}
$$

An expression for $\tilde{\mathbf{w}}$ which satisfies the traction-free conditions at $\theta=-\alpha$ and the jump conditions at $\theta=\psi$ is given by 


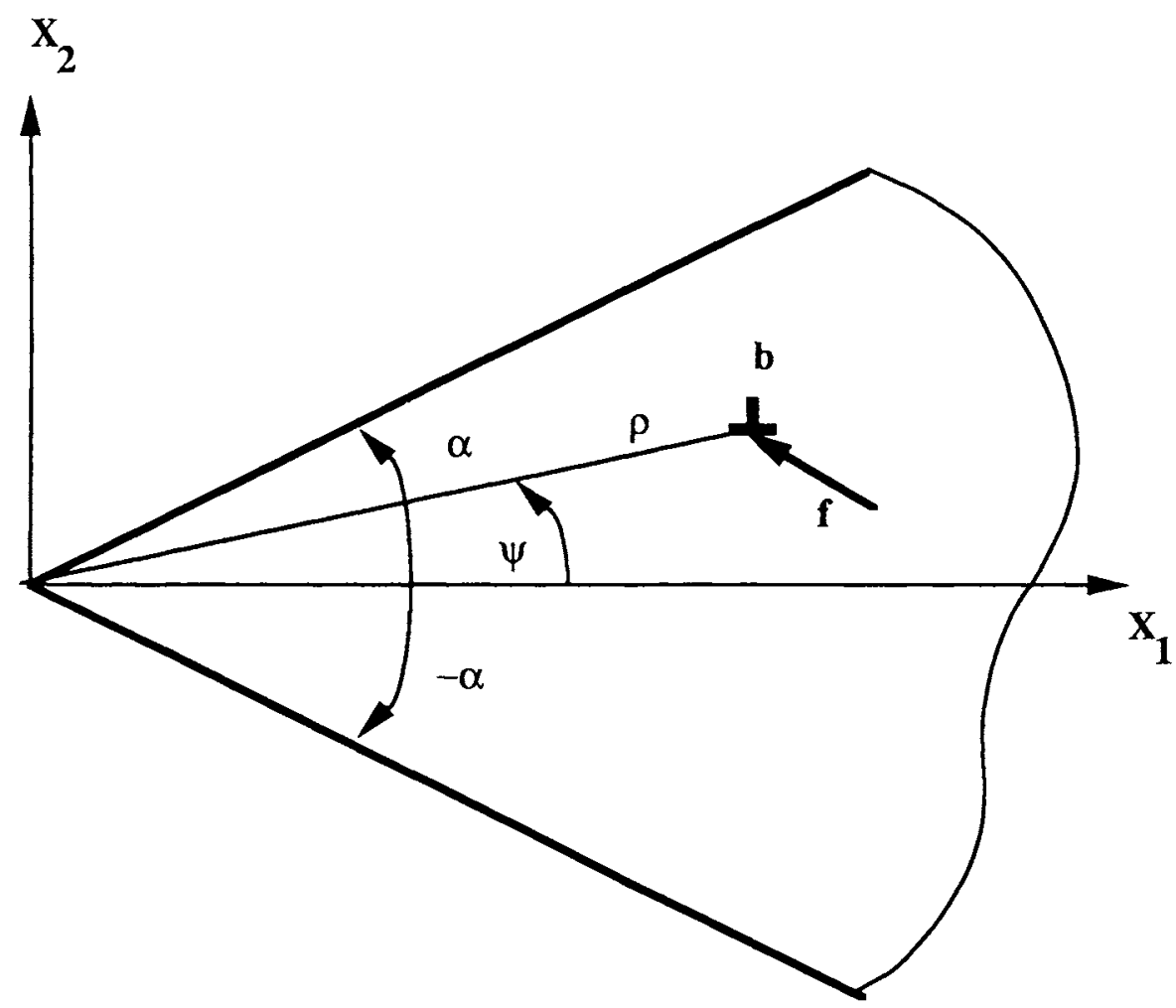

Fig. 1. A line force in an anisotropic elastic wedge.

$$
\tilde{\mathbf{w}}=\mathbf{G}(\theta,-\alpha ; \lambda)\left[\begin{array}{c}
\tilde{\mathbf{u}}(-\alpha) \\
\mathbf{0}
\end{array}\right]+\tilde{\mathbf{w}}^{*}
$$

where $\tilde{\mathbf{w}}^{*}$ are given by

$$
\tilde{\mathbf{w}}^{*}=H(\theta-\psi) \frac{\rho^{2}}{\lambda} \mathbf{G}(\theta, \psi ; \lambda) \mathbf{g}
$$

The vector $\tilde{\mathbf{u}}(-\alpha)$ in $\tilde{\mathbf{w}}$ is determined by the traction-free conditions at $\theta=\alpha$ as

$$
\tilde{\mathbf{u}}(-\alpha)=-\frac{\rho^{\lambda}}{\lambda} \mathbf{G}_{3}^{-1}(\alpha,-\alpha ; \lambda)\left(\mathbf{G}_{3}(\alpha, \psi ; \lambda) \mathbf{b}+\mathbf{G}_{1}^{T}(\alpha, \psi ; \lambda) \mathbf{f}\right)
$$

The solution $w$ in the physical domain is obtained by the Mellin inversion theorem as

$$
\mathbf{w}=\frac{1}{2 \pi i} \int_{c-i \infty}^{c+i \infty} r^{-\lambda} \tilde{\mathbf{w}} \mathrm{d} \lambda
$$

where $c$ is a proper real number.

\section{EIGENFUNCTION EXPANSIONS}

The integration contour in eqn (50) for $w$ can be closed by adding to the line of $\mathfrak{R}[\lambda]=c$ a semicircle of infinite radius to the right or the left in the complex $\lambda$ plane, depending on whether $r>\rho$ or $r<\rho$. Here $\mathfrak{R}[\lambda]$ stands for the real part of $\lambda$. The integral of eqn (50) can then be evaluated by the residues at the poles of $\tilde{\mathbf{w}}$ which are located in the 
regions $\mathfrak{R}[\lambda]>c$ for $r>\rho$ and $\mathfrak{R}[\lambda]<c$ for $r<\rho$. If the displacement is required to be bounded at the tip and the stress vanish at infinity, $c$ is chosen to be in the open strip $-\Re\left[\lambda_{1}\right]<\Re[\lambda]<0$, where $\lambda_{1}$ denotes the location of the pole of $\tilde{\boldsymbol{w}}$ with the smallest real part. Since the term $\tilde{w}^{*}$ in eqn (47) only has a simple pole at $\lambda=0$ where the residue is a constant, the term will be omitted in the following discussion for simplicity. Also to simplify the notation, $\mathbf{G}_{3}(\alpha,-\alpha ; \lambda)$ will be denoted as $\mathbf{G}_{3}(\lambda)$.

Consider the following eigenvalue problem :

$$
\mathbf{G}_{3}(\lambda) \mathbf{q}_{i}=\Delta_{i} \mathbf{q}_{i} \text { no sum on } i
$$

where $\Delta_{i}$ and $\mathbf{q}_{i}, i=1,2,3$, are the eigenvalues and eigenvectors of $\mathbf{G}_{3}(\lambda)$. As $\mathbf{G}_{3}(\lambda)$ is symmetric, the eigenvectors $\mathbf{q}_{i}$ and $\mathbf{q}_{j}$ can be normalized such that

$$
\mathbf{q}_{i}^{T} \mathbf{q}_{j}=\delta_{i j}
$$

and the eigenvalue $\Delta_{i}$ can be expressed as

$$
\Delta_{i}=\mathbf{q}_{i}^{T} \mathbf{G}_{3}(\lambda) \mathbf{q}_{i} \text { no sum on } i
$$

A crucial step in evaluating the residues is to express $G_{3}(\lambda)$ as the following spectral representation (Wu and Chiu, 1996):

$$
\mathbf{G}_{3}(\lambda)=\sum_{i=1}^{3} \Delta_{i} \mathbf{q}_{i} \mathbf{q}_{i}^{T}
$$

The inverse of $\mathbf{G}_{3}(\lambda)$ can then be represented as

$$
\mathbf{G}_{3}(\lambda)^{-1}=\sum_{i=1}^{3} \frac{1}{\Delta_{i}} \mathbf{q}_{i} \mathbf{q}_{i}^{T}
$$

Substitution of eqn (55) into eqn (49) yields

$$
\tilde{\mathbf{u}}(-\alpha)=-\sum_{i=1}^{3} \frac{\rho^{\lambda} c_{i}(\lambda)}{D_{i}} \mathbf{q}_{i}
$$

where

$$
\begin{gathered}
c_{i}(\lambda)=\mathbf{q}_{i}(\lambda)^{T}\left(\mathbf{G}_{3}(\alpha, \psi ; \lambda) \mathbf{b}+\mathbf{G}_{1}^{T}(\alpha, \psi ; \lambda) \mathbf{f}\right) \\
D_{i}=\lambda \Delta_{i}
\end{gathered}
$$

With eqn (56), $r^{-\lambda} \tilde{\mathbf{w}}$ of eqn (50) can be expressed as

$$
r^{-\lambda} \tilde{\mathbf{w}}=-\sum_{i=1}^{3} \frac{c_{i}}{D_{i}(\lambda)} \mathbf{W}(r, \theta ; \lambda)\left[\begin{array}{c}
\mathbf{q}_{i}(\lambda) \\
\mathbf{0}
\end{array}\right]
$$

where

$$
\mathbf{W}(r, \theta ; \lambda)=\sum_{\kappa=1}^{6}\left(\frac{r \zeta_{\kappa}(\theta)}{\rho \zeta(-\alpha)}\right)^{-\lambda} \boldsymbol{\xi}_{\kappa} \boldsymbol{\eta}_{\kappa}^{T}
$$

Equation (59) shows that the locations of the poles of $\tilde{\mathbf{w}}$ are the roots of 


$$
D_{i}(\lambda)=0
$$

Since

$$
\left|\mathbf{G}_{3}(\lambda)\right|=\Delta_{1} \Delta_{2} \Delta_{3}
$$

Equation (61) implies that at the poles $\mathbf{G}_{3}(\lambda)^{-1}$ is singular.

A pole of order $m$ exists at $\lambda=\lambda^{*}$ if $\lambda^{*}$ satisfies not only eqn (61) but

$$
D_{i}^{(j)}\left(\lambda^{*}\right)=j \Delta_{i}^{(j-1)}\left(\lambda^{*}\right)+\lambda \Delta_{i}^{(j)}\left(\lambda^{*}\right)=0, \quad j=1,2, \ldots, m-1
$$

where $D_{i}^{(j)}$ denotes the $j$-th derivative of $D_{i}$ with respect to $\lambda$. By taking $m$-th derivative of eqn (51) with respect to $\lambda$, setting $\Delta_{i}^{(j)}=0, j<m$, and pre-multiplying the resulting equation by $\mathbf{q}_{i}^{T}, \Delta_{i}^{(m)}$ can be expressed as

$$
\Delta_{i}^{(m)}=\mathbf{q}_{i}^{T}\left(\sum_{l=1}^{m} \frac{m !}{l !(m-l) !} \mathbf{G}_{3}^{(l)} \mathbf{q}_{i}^{(m-l)}\right)
$$

where

$$
\mathbf{G}_{3}^{(l)}(\lambda)=\sum_{\kappa=1}^{6} \log \left(\frac{\zeta_{\kappa}(-\alpha)}{\zeta_{\kappa}(\alpha)}\right)^{l}\left(\frac{\zeta_{\kappa}(\alpha)}{\zeta_{\kappa}(-\alpha)}\right)^{-\lambda} \mathbf{B}_{\kappa} \mathbf{B}_{\kappa}^{T}
$$

Note that $\Delta_{i}^{(m)}$ depends only on $\mathbf{q}_{i}$ and its derivative with respect to $\lambda$ up to $\mathbf{q}_{i}^{(m-1)}$. For example, for $m=1$,

$$
\Delta_{i}^{\prime}=\mathbf{q}_{i}^{T} \mathbf{G}_{3}^{\prime} \mathbf{q}_{i}
$$

Thus, once $\mathbf{q}_{i}$ is obtained, whether $\Delta_{i}^{\prime}$ vanishes or not can be readily checked.

It is obvious that if $\lambda^{*}$ is a pole of $\tilde{\boldsymbol{w}}$ so is $\bar{\lambda}^{*}$ and $\mathbf{q}\left(\bar{\lambda}^{*}\right)=\overline{\mathbf{q}\left(\lambda^{*}\right)}$. We show in the following that $-\lambda^{*}$ is also a pole. Using eqn (33), we have

$$
\mathbf{G}(-\lambda) \mathbf{G}(\lambda)\left[\begin{array}{c}
\mathbf{q}(\lambda) \\
\mathbf{0}
\end{array}\right]=\left[\begin{array}{c}
\mathbf{q}(\lambda) \\
\mathbf{0}
\end{array}\right]
$$

Equation (67) yields

$$
\mathbf{G}_{3}(-\lambda) \mathbf{G}_{1}(\lambda) \mathbf{q}(\lambda)+\Delta(\lambda) \mathbf{G}_{1}(-\lambda)^{T} \mathbf{q}(\lambda)=\mathbf{0}
$$

By pre-multiplying eqn (68) by $\mathbf{q}(-\lambda)^{T}$, we have

$$
\Delta(-\lambda)=-\Delta(\lambda) \frac{\mathbf{q}(-\lambda)^{T} \mathbf{G}_{1}(-\lambda)^{T} \mathbf{q}(\lambda)}{\mathbf{q}(-\lambda)^{T} \mathbf{G}_{1}(\lambda) \mathbf{q}(\lambda)}
$$

Since by definition $\Delta\left(\lambda^{*}\right)=0$, it follows from eqn (69) that $-\lambda^{*}$ is a root and from eqn (68)

$$
\mathbf{q}\left(-\lambda^{*}\right)=\gamma \mathbf{G}_{1}\left(\lambda^{*}\right) \mathbf{q}\left(\lambda^{*}\right)
$$

where $\gamma$ is a normalization factor such that $\mathbf{q}\left(-\lambda^{*}\right)^{T} \mathbf{q}\left(-\lambda^{*}\right)=1$. Furthermore, as 


$$
\left.\left(\frac{\partial^{j}}{\partial \lambda^{j}} \Delta(\lambda)\right)\right|_{\lambda=-\lambda^{*}}=\left.(-1)^{j}\left(\frac{\partial^{j}}{\partial \lambda^{j}} \Delta(-\lambda)\right)\right|_{\lambda=\lambda^{*}}
$$

Equation (69) implies that if $\lambda^{*}$ is order $m,-\lambda^{*}$ is also of order $m$.

It is obvious that $D_{i}(0)=0$ and $\lambda=0$ is always a pole. Moreover as $\Delta_{i}(0)=0$, and $D_{i}^{\prime}(0)=0, i=1,2,3, \lambda=0$ is of order two. It can be shown that $\lambda=1$ is also a pole regardless of the wedge angle. For $\lambda=1$, by setting $m=1, n=0$ in eqn (39), we have

$$
\mathbf{G}_{3}(1)=\mathbf{N}_{3}(-\alpha, \alpha)
$$

From eqn (42), the corresponding eigenvector is given by

$$
\mathbf{q}(1)=\mathbf{m}(\alpha)
$$

It is remarkable that the eigenvector $\mathbf{q}(1)$ is independent of the elastic constants and is perpendicular to the wedge face at $\theta=\alpha$. From eqn (39) with $m=n=1$, the matrix $\mathbf{G}_{3}^{\prime}(1)$ can be expressed as

$$
\begin{aligned}
\mathbf{G}_{3}^{\prime}(1) & =-\sum_{\kappa=1}^{6} \frac{\zeta_{\kappa}(-\alpha)}{\zeta_{\kappa}(\alpha)} \log \left(\frac{\zeta_{\kappa}(\alpha)}{\zeta_{\kappa}(-\alpha)}\right) \mathbf{B}_{\kappa} \mathbf{B}_{\kappa}^{T} \\
& =\pi\left[\mathbf{N}_{3}(-\alpha, \alpha) \mathbf{N}_{1}(-\alpha, \alpha)+\tilde{\mathbf{N}}_{1}^{T}(-\alpha, \alpha) \mathbf{N}_{3}(-\alpha, \alpha)\right]
\end{aligned}
$$

Substituting eqns (73) and (74) into eqn (66), we have

$$
\Delta^{\prime}(1)=-\pi \mathbf{m}(\alpha)^{T} \tilde{\mathbf{N}}_{3}(\alpha,-\alpha) \mathbf{m}(-\alpha)
$$

where eqn (42) has been used. Let $\alpha^{*}$ be the critical wedge angle such that

$$
\mathbf{m}\left(\alpha^{*}\right)^{T} \tilde{\mathbf{N}}_{3}\left(\alpha^{*},-\alpha^{*}\right) \mathbf{m}\left(-\alpha^{*}\right)=0
$$

The pole at $\lambda=1$ is of order two when $\alpha=\alpha^{*}$ and is simple otherwise. Equation (76) has also been given by (Hwu and Ting, 1990) for a wedge subject to a couple at the tip.

By summing the residues at the poles, $w$ for $r>\rho$ can be expressed as

$$
\mathbf{w}=\mathbf{w}_{0}+\sum_{n=1}^{\infty} \mathbf{w}_{n}
$$

and for $r<\rho$

$$
\mathbf{w}=\sum_{n=1}^{\infty} \mathbf{w}_{-n}
$$

where $-\mathbf{w}_{0},-\mathbf{w}_{n}$, and $\mathbf{w}_{-n}$ are the residues of eqn (59) at $\lambda=0, \lambda_{n}$, and $-\lambda_{n}$, respectively. Each term in eqn (77) or eqn (78) satisfies the traction-free conditions on the wedge faces and thus, eqns (77) and (78) are actually eigenfunction expansions of $\mathbf{w}$ for $r>\rho$ and $r<\rho$, respectively.

Consider first the general terms in the eigenfunction expansions for the special angles $\alpha=m \pi / 2, m=1,2$. The case for $m=1$ corresponds to a half-space and $m=2$ to a semiinfinite crack. The matrix $\mathbf{G}_{3}(\lambda)$ in these cases can be simplified as

$$
\mathbf{G}_{3}(\lambda)=\sin (m \pi \lambda) \mathbf{L}
$$

where $L$ is a real and positive-definite matrix defined by (Chadwick and Smith, 1977) 


$$
\mathbf{L}=-2 i \sum_{\kappa=1}^{3} \mathbf{B}_{\kappa} \mathbf{B}_{\kappa}^{T}
$$

It follows that $\Delta_{i}=\sin (m \pi \lambda) L_{i}, i=1,2,3, L_{i}>0$ being the eigenvalues of $\mathbf{L}$ and that the eigenvectors $\mathbf{q}_{i}$ are those of $\mathbf{L}$. It is apparent that $\lambda_{n}=n / m, n$ being an integer, is a simple pole regardless of the elastic constants. Evaluation of the residue of eqn (59) at $\lambda_{n}$ yields

$$
\begin{gathered}
\mathbf{w}_{n}=\frac{(-1)^{n}}{n \pi}\left(\frac{\rho}{r}\right)^{n / m} \mathbf{G}\left(\theta,-\frac{m \pi}{2} ; \frac{n}{m}\right)\left[\begin{array}{c}
\mathbf{d}\left(\frac{m \pi}{2}, \psi ; \frac{n}{m}\right) \\
\mathbf{0}
\end{array}\right] \\
\mathbf{w}_{-n}=\frac{(-1)^{n}}{n \pi}\left(\frac{r}{\rho}\right)^{n / m} \mathbf{G}\left(\theta,-\frac{m \pi}{2} ;-\frac{n}{m}\right)\left[\begin{array}{c}
\mathbf{d}\left(\frac{m \pi}{2}, \psi ;-\frac{n}{m}\right) \\
\mathbf{0}
\end{array}\right]
\end{gathered}
$$

where

$$
\mathbf{d}\left(\frac{m \pi}{2}, \psi ; \frac{n}{m}\right)=\mathbf{L}^{-1}\left(\mathbf{G}_{3}\left(\frac{m \pi}{2}, \psi ; \frac{n}{m}\right) \mathbf{b}+\mathbf{G}_{1}^{T}\left(\frac{m \pi}{2}, \psi ; \frac{n}{m}\right) \mathbf{f}\right)
$$

By substituting eqn (98) with $\alpha=m \pi / 2, m=1,2$ and eqn (81) into eqn (77) or eqn (82) into eqn (78), the resulting series can be summed to yield the following closed form expressions :

$$
\begin{aligned}
& \mathbf{u}=\frac{1}{\pi}\left(\mathfrak{I}\left[\mathbf{A C}_{m} \mathbf{h}\right]+\mathfrak{I}\left[\mathbf{A D}_{m} \overline{\mathbf{h}}\right]\right) \\
& \phi=\frac{1}{\pi}\left(\mathfrak{I}\left[\mathbf{B C}_{m} \mathbf{h}\right]+\mathfrak{I}\left[\mathbf{B D}_{m} \overline{\mathbf{h}}\right]\right)
\end{aligned}
$$

where $\mathbf{A}=\left[\mathbf{A}_{1}, \mathbf{A}_{2}, \mathbf{A}_{3}\right], \mathbf{B}=\left[\mathbf{B}_{1}, \mathbf{B}_{2}, \mathbf{B}_{3}\right], \mathbf{h}=\mathbf{A}^{T_{\mathbf{f}}}+\mathbf{B}^{T} \mathbf{b}, \mathbf{C}_{1}$ and $\mathbf{D}_{1}$ for a half-space are given by

$$
\begin{gathered}
\left(\mathbf{C}_{1}\right)_{i j}=\log \left(r \zeta_{i}(\theta)-\rho \zeta_{i}(\psi)\right) \delta_{i j} \\
\left(\mathbf{D}_{1}\right)_{i j}=\log \left(\frac{r \zeta_{i}(\theta) \bar{p}_{j}}{p_{i}}-\rho \bar{\zeta}_{i}(\psi)\right) Y_{i j}
\end{gathered}
$$

and $\mathbf{C}_{2}$ and $\mathbf{D}_{2}$ for a semi-infinite crack are

$$
\begin{gathered}
\left(\mathbf{C}_{2}\right)_{i j}=\log \left(\sqrt{r \zeta_{i}(\theta)}-\sqrt{\rho \zeta_{i}(\psi)}\right) \delta_{i j} \\
\left(\mathbf{D}_{2}\right)_{i j}=\log \left(-\sqrt{r \zeta_{i}(\theta)}-\sqrt{\rho \bar{\zeta}_{j}(\psi)}\right) Y_{i j}
\end{gathered}
$$

with $\mathbf{Y}=\mathbf{B}^{-1} \overline{\mathbf{B}}$. The form of the solution for a half-space is also given by Ting (1996).

For simplicity it is assumed that for $\alpha \neq \pi / 2, \pi$, only one of the eigenvalues $\Delta_{i}$ vanishes at the pole $\lambda_{n}, n>0$. The eigenvalue is simply denoted by $\Delta\left(\lambda_{n}\right)$ and the corresponding eigenvector by $\mathbf{q}\left(\lambda_{n}\right)$. From eqn (59), the terms $\mathbf{w}_{n}, n \geqslant 0$ in eqn (77) are given by 


$$
\mathbf{w}_{n}=\left.\frac{1}{(m-1) !} \frac{\partial^{m-1}}{\partial \lambda^{m-1}}\left(\left(\lambda-\lambda_{n}\right)^{m} \frac{c(\lambda)}{D(\lambda)} \mathbf{W}(r, \theta ; \lambda)\left[\begin{array}{c}
\mathbf{q}(\lambda) \\
\mathbf{0}
\end{array}\right]\right)\right|_{\lambda=\lambda_{n}}
$$

if the pole at $\lambda=\lambda_{n}$ is of order $m$. While the terms $\mathbf{w}_{-n}, n>0$ in eqn (78) are obtained by

$$
\mathbf{w}_{-n}=-\left.\frac{1}{(m-1) !} \frac{\partial^{m-1}}{\partial \lambda^{m-1}}\left(\left(\lambda+\lambda_{n}\right)^{m} \frac{c(\lambda)}{D(\lambda)} \mathbf{W}(r, \theta ; \lambda)\left[\begin{array}{c}
\mathbf{q}(\lambda) \\
\mathbf{0}
\end{array}\right]\right)\right|_{\lambda=-\lambda_{n}}
$$

If $\lambda_{n}$ is a simple pole, eqns (90) and (91) reduce to

$$
\begin{gathered}
\mathbf{w}_{n}=\frac{c\left(\lambda_{n}\right)}{\lambda_{n} \Delta^{\prime}\left(\lambda_{n}\right)} \mathbf{W}\left(r, \theta ; \lambda_{n}\right)\left[\begin{array}{c}
\mathbf{q}\left(\lambda_{n}\right) \\
0
\end{array}\right] \\
\mathbf{w}_{-n}\left(\lambda_{n}\right)=-\mathbf{w}_{n}\left(-\lambda_{n}\right)
\end{gathered}
$$

\section{ASYMPTOTIC FIELDS}

For definiteness let $\lambda_{n}$ in eqns (77) and (78) be arranged in increasing real parts. In this section the leading terms $\mathbf{w}_{0}$ and $\mathbf{w}_{1}$ as $r \rightarrow \infty$ and $\mathbf{w}_{-1}$ as $r \rightarrow 0$ are discussed.

From preceding discussion $\lambda=0$ is a pole of order two. The expression for $\mathbf{w}_{0}$ derived from eqn (90) with $m=2$ is given by

$$
\mathbf{w}_{0}=\sum_{i=1}^{3} \frac{\mathbf{q}_{i}(0)^{T} \mathbf{f}}{\Delta_{i}^{\prime}(0)} \mathbf{W}^{\prime}(r, \theta ; 0)\left[\begin{array}{c}
\mathbf{q}_{i}(0) \\
\mathbf{0}
\end{array}\right]
$$

where

$$
\mathbf{W}^{\prime}(r, \theta ; 0)=-\sum_{\kappa=1}^{6} \log \left(\frac{r \zeta_{\kappa}(\theta)}{\rho \zeta_{\kappa}(-\alpha)}\right) \boldsymbol{\xi}_{\kappa} \boldsymbol{\eta}_{\kappa}^{T}
$$

In eqn (94) certain constant terms, which are related to rigid body translations are omitted. By differentiating eqn (51) with respect to $\lambda$ at $\lambda=0$, we have

$$
\mathbf{G}_{3}^{\prime}(0) \mathbf{q}_{i}=\Delta_{i}^{\prime}(0) \mathbf{q}_{i}
$$

and

$$
\mathbf{G}_{3}^{\prime-1}(0)=\sum_{i=1}^{3} \frac{\mathbf{q}_{i} \mathbf{q}_{i}^{T}}{\Delta_{i}^{\prime}(0)}
$$

With eqn (97), eqn (94) can be rewritten as

$$
\mathbf{w}_{0}=\mathbf{W}^{\prime}(r, \theta ; 0)\left[\begin{array}{c}
\mathbf{G}_{3}^{\prime}(0)^{-1} \mathbf{f} \\
\mathbf{0}
\end{array}\right]
$$

Equation (98) represents the solution for a force $\mathbf{f}$ acting on the tip (Barnett and Lothe, 1975).

Since $\lambda=1$ is always a pole, $\lambda_{1}$ is either less than or equal to one. If $\lambda_{1}<1, \mathbf{w}_{1}$ and $\mathbf{w}_{-1}$ are simply given by eqn (90) and eqn (91), respectively, with $n=1$. In this case the stresses corresponding to $\mathbf{w}_{-1}$ are proportional to $r^{\lambda_{1}-1}$ and are singular at the tip. The result has been derived by Wu and Chang (1993) using a different approach. 
If $\lambda_{1}=1$ and $\Delta^{\prime}(1) \neq 0$ or $\alpha \neq \alpha^{*}$, $\alpha^{*}$ being the critical angle determined by eqn (76), the pole is simple. The far field $\mathbf{w}_{1}$ is given by eqn (92) as

$$
\mathbf{w}_{1}=\frac{M}{\Delta^{\prime}(1) r} \mathbf{N}(-\alpha, \theta)\left[\begin{array}{c}
\mathbf{m}(\alpha) \\
\mathbf{0}
\end{array}\right]
$$

where $\Delta^{\prime}(1)$ is given by eqn (75) and $M$ is the moment exerted by the force with respect to the tip:

$$
M=\rho \mathbf{m}(\psi)^{T} \mathbf{f}
$$

Equation (99) has been derived by Hwu and Ting (1990). The near field $\mathbf{w}_{-1}$, obtained by eqn (93), represents a rigid body rotation $\Omega$ given by

$$
\Omega=\frac{1}{\rho \Delta^{\prime}(1)} \mathbf{m}^{T}(\alpha)\left(\mathbf{N}_{3}^{T}(-\alpha, \psi) \mathbf{b}+\mathbf{N}_{1}^{T}(-\alpha, \psi) \mathbf{f}\right)
$$

If $\lambda_{1}=1$ and $\Delta^{\prime}(1)=0$ or $\alpha=\alpha^{*}$, the pole is of order two. The far field $w_{1}$ can be derived from eqn (90) with $m=2$. The result can be expressed as

$$
\mathbf{w}_{1}=\mathbf{w}_{11}+\mathbf{w}_{12}
$$

where

$$
\begin{gathered}
\mathbf{w}_{11}=\frac{\rho}{r}\left(\frac{c^{\prime}+c \log (\rho / r)}{h}-\frac{h^{\prime} c}{h^{2}}\right) \mathbf{G}\left(-\alpha^{*}, \theta ; 1\right)\left[\begin{array}{c}
\mathbf{m}\left(\alpha^{*}\right) \\
0
\end{array}\right] \\
\mathbf{w}_{12}=\frac{c r}{h r}\left(\mathbf{G}^{\prime}\left(-\alpha^{*}, \theta ; 1\right)\left[\begin{array}{c}
\mathbf{m}\left(\alpha^{*}\right) \\
0
\end{array}\right]+\mathbf{G}\left(-\alpha^{*}, \theta ; 1\right)\left[\begin{array}{c}
\mathbf{q}^{\prime}(1) \\
\mathbf{0}
\end{array}\right]\right)
\end{gathered}
$$

Here

$$
\begin{aligned}
c= & \mathbf{m}(\psi)^{T} \mathbf{F} \\
c^{\prime}= & \left(\mathbf{q}^{\prime}(1)^{T} \mathbf{N}_{1}^{T}\left(\psi, \alpha^{*}\right)-\pi \mathbf{m}(\psi)^{T} \overline{\mathbf{N}}_{1}\left(\alpha^{*}, \psi\right)\right) \mathbf{F} \\
& +\left(\mathbf{q}^{\prime}(1)^{T} \mathbf{N}_{3}^{T}\left(\psi, \alpha^{*}\right)-\pi \mathbf{m}(\psi)^{T} \overline{\mathbf{N}}_{3}\left(\alpha^{*}, \psi\right)\right) \mathbf{b} \\
h= & \frac{1}{2} \Delta^{\prime \prime}(1) \\
h^{\prime}= & \frac{1}{2} \Delta^{\prime \prime}(1)+\frac{1}{6} \Delta^{\prime \prime \prime}(1) \\
\mathbf{G}^{\prime}\left(-\alpha^{*}, \theta ; 1\right)= & \pi \tilde{\mathbf{N}}\left(-\alpha^{*}, \theta\right) \mathbf{N}\left(-\alpha^{*}, \theta\right) \\
\mathbf{q}^{\prime}(1)= & \pi \mathbf{N}_{3}\left(-\alpha^{*}, \alpha^{*}\right)^{\langle-1\rangle} \overline{\mathbf{N}}_{3}\left(\alpha^{*},-\alpha^{*}\right) \mathbf{m}\left(-\alpha^{*}\right)
\end{aligned}
$$

and $\mathbf{N}_{3}\left(-\alpha^{*}, \alpha^{*}\right)^{\langle-1\rangle}$ is defined as

$$
\mathbf{N}_{3}\left(-\alpha^{*}, \alpha^{*}\right)^{\langle-1\rangle}=\frac{1}{\Delta_{2}} \mathbf{q}_{2} \mathbf{q}_{2}^{T}+\frac{1}{\Delta_{3}} \mathbf{q}_{3} \mathbf{q}_{3}^{T}
$$

where $\Delta_{i}, i=2,3$ are two nonzero eigenvalues of $\mathbf{N}_{3}\left(-\alpha^{*}, \alpha^{*}\right)$ and $\mathbf{q}_{i}, i=2,3$ are the corresponding eigenvectors. It can be shown that the moment exerted by the force is balanced solely by that produced by $\mathbf{w}_{12}$. In fact $\mathbf{w}_{11}$ contains a term multiplied by $\log (\rho)$ and is not related to the ordinary moment of force. Equation (102) is also applicable to isotropic material. Indeed the expressions for the corresponding stresses have been checked 
to agree with those derived by Sternberg and Koiter (1958). The near field $\mathbf{w}_{-1}$ can be obtained from eqn (91) with $m=2$. The result is identical with that derived by Wu and Chang (1993) and will not be reproduced here.

\section{CONCLUDING REMARKS}

The complete solution due to a line force or a line dislocation in an anisotropic wedge is derived. With Stroh's formalism the solution is expressed in an explicit and compact form. Several solutions in the existing literature are included in the solution obtained here. In particular the solutions for a wedge subject to a force (Barnett and Lothe, 1975) and a couple (Ting, 1988; Hwu and Ting, 1990) at the tip appear as terms for $\lambda=0$ and $\lambda=1$, respectively, in the eigenfunction series of the present solution. It is shown that the critical wedge angle as discussed by Hwu and Ting (1990) is the one that gives rise to a double pole at $\lambda=1$.

\section{REFERENCES}

Barnett, D. M. and Lothe, J. (1975) Line force loadings on anisotropic half-spaces and wedges. Phys. Norv. 8, $13-22$.

Bogy, D. B. (1972) The plane solution for anisotropic elastic wedges under normal and shear loading. $A S M E$ Journal of Applied Mechanics 39, 1103-1109.

Chadwick, P. and Smith, G. D. (1977) Foundations of the theory of surface waves in anisotropic materials. Advances in Applied Mechanics 17, 303-376.

Chou, Y. T. (1966) On dislocation-boundary interaction in an anisotropic aggregate. Phys. Status Solidi B15, $123-127$.

Eshelby, J. D., Read, W. T. and Shockley, W. (1953) Anisotropic elasticity with applications to dislocation theory. Acta Metallica 1, 251-259.

Head, A. K. (1953) Interaction of dislocations and boundaries. Philosophical Magazine 44, 92-94.

Head, A. K. (1965) The dislocation image force in cubic polycrystals. Phys. Status Solidi B10, $481-484$.

Hoffman, K. and Kunze, R. (1961) Linear Algebra. Prentice-Hall, New Jersey.

Hwu, C. and Ting, T. C. T. (1990) Solution for the anisotropic elastic wedge at critical angles. Journal of Elasticity 24, $1-20$

Malen, K. (1971) A unified six-dimensional treatment of elastic Green's functions and dislocations. Phys. Status Solidi B44, 661-672.

Malen, K. and Lothe, J. (1970) Explicit expressions for dislocation derivatives. Phys. Status Solidi B39, 287-296.

Rizzo, R. (1967) An integral equation approach to boundary value problems in classical elastostatics. Quarterly Journal of Applied Mathematics 25, 83-95.

Sternberg, E. and Koiter, W. T. (1958) The wedge under a concentrated couple : a paradox in the two-dimensional theory of elasticity. ASME Journal of Applied Mechanics 25, 575-581.

Stroh, A. N. (1958) Dislocations and cracks in anisotropic elasticity. Philosophical Magazine 7, 625-646.

Ting, T. C. T. (1988) The anisotropic elastic wedge under a concentrated couple. Quarterly Journal of the Mechanics of Applied Mathematics 41, 563-578.

Ting, T. C. T. (1996) Anisotropic Elasticity-Theory and Applications. Oxford University Press, New York.

Tucker, M. O. (1969) Plane boundaries and straight dislocations in elastically anisotropic materials. Philosophical Magazine 19, 1141-1159.

Willis, J. R. (1970) Stress field produced by dislocations in anisotropic media. Philosophical Magazine 21, 931949.

Wu, K.-C. and Chang, F.-T. (1993) Near-tip fields in a notched body with dislocations and body forces. $A S M E$ Journal of Applied Mechanics 60, 936-941.

Wu, K.-C. and Chiu, Y.-T. (1996) Analysis for elastic strips under concentrated loads. ASME Journal of Applied Mechanics, submitted.

Wu, K.-C., Chiu, Y.-T. and Hwu, Z.-H. (1992) A new boundary integral equation formulation for linear elastic solids. ASME Journal of Applied Mechanics 59, 344-348. 